ArtefaCToS. Revista de estudios de la ciencia y la tecnología

eISSN: $1989-3612$

Vol. 7, No. 2 (2018), 2a Época, 165-184

DOI: http://dx.doi.org/10.14201/art201872165184

\title{
Hacia una noción más robusta de adecuación empírica
}

\author{
Towards a More Robust Notion of Empirical Adequacy
}

María CAAMAÑO ALEGRE

Universidad de Valladolid

mariac@fyl.uva.es

Recibido: 11/09/2018. Revisado: 19/09/2018. Aceptado: 19/09/2018

\section{Resumen}

En este artículo se propone un criterio de adecuación empírica orientado a la superación de ciertas limitaciones que afectan a las concepciones tradicionales, consecuencialistas del éxito empírico de las teorías. En el criterio propuesto cobran especial importancia los siguientes aspectos: a) la resolución de anomalías no refutatorias, b) la superioridad de la predicción frente a la acomodación, y c) el uso limitado de hipótesis ad hoc.

Palabras clave: adecuación empírica; predicción; acomodación; anomalías no refutatorias; hipótesis ad hoc.

\begin{abstract}
This paper offers a criterion of empirical adequacy aimed at overcoming certain limitations that affect traditional, consequentialist accounts of the empirical success of theories. The suggested criterion emphasizes the importance of the following aspects: a) the resolution of non-refuting anomalies, $b$ ) the superiority of prediction over accommodation, and c) the limited use of ad hoc hypotheses.
\end{abstract}

Keywords: empirical adequacy; prediction; accommodation; non-refuting anomalies; ad hoc hypothesis. 


\section{Introducción}

El problema de cómo entender la adecuación empírica de las teorías se remonta a la antigüedad, manteniendo Platón y Aristóteles concepciones distintas acerca de lo que debía significar, para los astrónomos, "salvar los fenómenos" (Duhem 1908/2015). Tal vez como una herencia del instrumentalismo platónico en lo que se refería al trabajo empírico de los astrónomos, en la filosofía de la ciencia contemporánea suele asociarse a autores considerados "instrumentalistas", como Bas van Fraassen y Larry Laudan, el énfasis en la adecuación empírica como principal criterio de evaluación de teorías. Sin embargo, el realismo crítico de Karl Popper pone igualmente en primer plano la exigencia de adecuación empírica, al proponer el desiderátum metodológico de maximizar, a la vez, las posibilidades de descubrimiento de la verdad y de escrutinio empírico de las conjeturas propuestas para tal descubrimiento. Este desiderátum nos remite a un amplio campo de problemas metodológicos conectados con la insistencia popperiana y lakatosiana en el valor de las predicciones novedosas y heterogéneas frente a la mera acumulación de predicciones del mismo tipo. El reto que surge a partir del reconocimiento de la importancia de las predicciones sorprendentes es, en última instancia, el de proponer criterios para determinar, no sólo la cantidad sino también la calidad de la evidencia en favor de una conjetura.

Este trabajo se centra en una parte fundamental de los criterios de evaluación de las teorías científicas, a saber, los criterios de adecuación empírica. Es decir, se trata de identificar los factores principales que determinan la superioridad de una teoría frente a otra en lo que respecta a su adecuación empírica. Para ello, se tendrán en cuenta ciertos requisitos propuestos de manera independiente, entre otros, por Larry Laudan (1981, 1995, 2000), Peter Lipton (1991/2004), Christopher Hitchcok junto con Elliot Sober (2004), Paul Thagard (1978), Martin Carrier (1988) y Jarrett Leplin (1975) para una evaluación del éxito empírico de las teorías más sensible a aspectos cualitativos tradicionalmente desatendidos desde la filosofía de la ciencia.

En lo que sigue, pondré primero de relieve las limitaciones que afectan a la concepción tradicional de la adecuación empírica, proponiendo a continuación un criterio esquemático para la evaluación comparativa de teorías en lo que respecta a su éxito empírico. El criterio incluirá condiciones que reflejen la especial importancia de: a) la resolución de anomalías no refutatorias, b) la superioridad de la predicción frente a la acomodación, y c) el uso limitado de hipótesis ad hoc.

Se dejarán al margen la cuestión acerca de si el éxito empírico de una teoría implica algún tipo de aproximación de la verdad, o si proporciona una base para una concepción realista de las teorías científicas. Dado que los argumentos en favor de estas ideas se basan, en gran medida, en consideraciones 
relativas al éxito empírico, la aclaración de éste último resulta importante independientemente del punto de vista que se adopte con respecto a la cuestión del realismo.

\section{La insuficiencia de los criterios cuantitativos de adecuación empírica}

En un esclarecedor análisis de la expresión 'saving the phenomena', L. Laudan $(2002,168-9)$ distingue tres sentidos fundamentales en los que ha sido usada dentro del contexto filosófico: como corrección observacional ( $a$ la van Fraassen), implicando, por tanto, que todas las consecuencias observacionales de una teoría son verdaderas, como explicación de todos los hechos importantes conocidos dentro de un determinado dominio de investigación (según la concepción de Platón), y, finalmente, como corrección observacional más capacidad explicativa para dar cuenta de todos los fenómenos ya explicados por teorías precedentes. Laudan argumenta convincentemente que, si entendemos la adecuación empírica exclusivamente en el primer sentido (el de corrección observacional), entonces difícilmente podremos discriminar entre teorías con mayor o menor éxito empírico, puesto que teorías de muy distinto alcance podrían ser todas ellas observacionalmente correctas. Por otra parte, como ya se señaló, los criterios tradicionales para el éxito empírico giraron principalmente en torno al número de aplicaciones exitosas de una teoría -que, desde la perspectiva de las teorías (Popper, 1962), equivale al número de consecuencias empíricas verdaderas de una teoría, $\mathrm{y}$, desde un enfoque modelo-teórico (Moulines, 2000), al número de fenómenos incorporados con éxito en los modelos teóricos a través de las subestructuras empíricas de esos modelos. Al caracterizar una teoría $\left(\mathrm{T}_{2}\right)$ como más exitosa que otra teoría $\left(\mathrm{T}_{1}\right)$, las concepciones tradicionales de éxito teórico $(\mathrm{S})$ generalmente se comprometen con la siguiente condición meramente cuantitativa: $S\left(T_{1}\right) \subset$ $\mathrm{S}\left(\mathrm{T}_{2}\right)$, algunas veces complementada con la condición kuhniana de que $\mathrm{S}\left(\mathrm{T}_{2}\right)$ incluya algunas de las anomalías más recalcitrantes de $\mathrm{T}_{1}$.

Sin embargo, esta explicación puramente cuantitativa del éxito teórico, incluso si se complementa con el supuesto de que existe un criterio epistemológico-pragmático interteórico para priorizar ciertas aplicaciones intencionales cuya posible extensión a modelos es de especial interés, no es lo suficientemente revelador como análisis del éxito empírico de las teorías. Un problema importante tiene que ver con el hecho de que los requisitos establecidos sobre el éxito de las teorías en competencia son meramente cuantitativos $\mathrm{y}$, por esta razón, insensibles, tanto a la importancia empírica de las aplicaciones intencionales cubiertas por las teorías, como al valor explicativo de éstas. Con respecto al primero, cabe mencionar la especial relevancia dada a las predicciones novedosas e inesperadas o a las relativas a fenómenos prominentes que a las que la teoría inicialmente no pretendía aplicarse. Parece plausible pensar que, incluso si una teoría no satisface la condición mencionada en el último 
párrafo, podría todavía considerarse como más exitosa que su competidora en caso de que sólo la primera proporcionara predicciones inesperadas y una explicación para fenómenos sobresalientes previamente ignorados. La teoría de la relatividad puede ilustrar este tipo de éxito, ya que se consideró exitosa, al menos en parte por estas razones, incluso antes de que pudiera confirmarse en la misma medida que lo había sido la mecánica newtoniana. También puede haber casos (como las teorías ptolemaica y copernicana) en los que las teorías rivales no difieren en su éxito cuantitativo (al menos durante cierto período de tiempo) y, sin embargo, una de ellas se considera explicativamente superior a la otra, además de empíricamente mejor respaldada. Las dos condiciones anteriores no son aplicables aquí, y sin embargo parece haber una clara diferencia en el éxito entre las dos teorías. Antes de Galileo, la astronomía ptolemaica y copernicana podían explicar aproximadamente los mismos fenómenos. Sin embargo, la segunda fue considerada como una mejor explicación, no sólo por su mayor simplicidad y capacidad unificadora, sino también por su carácter menos ad hoc. Otro problema que afecta al punto de vista tradicional se refiere a la escasa atención prestada al alcance, es decir, a la generalidad o la riqueza informativa de una teoría como elemento de éxito. En otras palabras, el requisito cuantitativo se puede cumplir sin que el alcance o dominio de aplicación exitoso de una teoría cambie significativamente.

Las observaciones anteriores sugieren que algunas preguntas importantes siguen sin respuesta en las explicaciones tradicionales sobre el éxito empírico de las teorías. Estas preguntas apuntan a la necesidad de refinamientos en la forma de varios requisitos cualitativos, que deberían centrarse especialmente en cuestiones relacionadas con el alcance de una teoría, la independencia de la evidencia y la adhocidad. Más en particular, tales requisitos se referirán a: a) la resolución de anomalías no refutatorias (Laudan, 2000, 166-7), b) la superioridad de la predicción frente a la acomodación (Lipton, 1991/2004, 68), y c) el uso limitado de hipótesis auxiliares no corroboradas (Thagard, 1978, 86-9). Nótese la relevancia especial que todas las características anteriores tienen para la cuestión de la adecuación empírica, y que se hace evidente en el hecho de que a) está directamente conectada tanto con la adecuación empírica como con la riqueza informativa o de contenido; b) está relacionada con ésta e indirectamente con la adecuación empírica (posibilidad de aumentar la adecuación empírica aumentando la riqueza de contenido); y c) está indirectamente relacionada con la adecuación empírica de una teoría, ya que está directamente relacionada con la adecuación empírica de las hipótesis auxiliares asociadas a ella.

En la sección 4 se esboza una respuesta a estos problemas a partir de la caracterización de una noción más robusta de adecuación empírica. Pero antes me detendré en aquellos factores habitualmente ignorados que algunos de los autores ya mencionados consideran determinantes para la evaluación del éxito empírico de las teorías. 


\section{Aspectos cualitativos implicados en la adecuación empírica}

Tras haber analizado algunas de las principales deficiencias de los enfoques tradicionales y consecuencialistas de la adecuación empírica, me centraré en algunas de las propuestas que pueden resultar de mayor utilidad para fortalecer el criterio de éxito empírico para las teorías. Abordaré en primer lugar, los problemas relativos al alcance de una teoría, para luego centrarme en la cuestión de la superioridad de la predicción frente a la acomodación y finalmente en el problema de la adhocidad.

\subsection{La relevancia empírica de las anomalías no refutatorias}

Laudan introduce la noción de anomalía no refutatoria en su obra de 1977 Progress and its Problems, donde caracteriza las anomalías en general como "problemas empíricos que plantean dudas razonables sobre la adecuación empírica de una teoría", una vez que otra teoría los ha solucionado" (1977, $28,30)$. Según él, las anomalías no refutatorias, a diferencia de las refutatorias, no implican ninguna incompatibilidad lógica entre las consecuencias empíricas de la teoría, por una parte, y las afirmaciones verificadas sobre hechos empíricos, por otra (1977, 27-29). Más bien implican la incapacidad de una teoría para dar cuenta de cierto tipo de fenómenos empíricos llamativos cuya descripción es consistente con todo lo establecido por la teoría. Como dice Laudan:

Such non-refuting anomalies typically arise when one theory is compatible with, but offers no solution to (or explanation of), certain phenomena for which some its rivals can give an account. It was my claim that the scientific methodologies of logical empiricism had not recognized this historically significant form of anomaly. (Laudan, 1981, 618)

Las anomalías no refutatorias, por lo tanto, no apuntan principalmente a ningún error en la forma en que una teoría explica los fenómenos, sino más bien al carácter incompleto de la teoría. Los casos típicos en los que esto sucede son señalados por Laudan al desarrollar la noción de anomalías no refutatorias en el contexto de su argumentación a favor de la importancia de la completitud como virtud teórica. En su trabajo de 1977 menciona dos casos: la incapacidad de la cinemática pregalileana para explicar las características matemáticas del movimiento pendular, es decir, la ausencia de predicciones para la geometría de un peso en movimiento y la falta de explicación de la mecánica newtoniana para la coplanaridad y dirección común de las órbitas planetarias, que habían sido acomodadas en las astronomías kepleriana y cartesiana $(1977,29)$. Algunos otros ejemplos se agregan en su texto de 2000; por ejemplo, el encaje del contorno de los continentes, para lo cual las teorías 
que incluían el supuesto de la estabilidad de los continentes no ofrecieron ninguna explicación, o el fenómeno de la radiación residual de fondo, que permaneció inexplicada por la cosmología del estado estacionario $(1977,167)$.

La concepción de las anomalías de Laudan, fuertemente influenciada por la kuhniana (1962/1970, 52-65), ${ }^{1}$ incluye la idea de que las anomalías sólo pueden considerarse como afectando a cierta teoría una vez que otra teoría ha sido capaz de resolverlas. ${ }^{2}$ Aquí se favorece en cambio una noción más amplia de anomalía. Según esta caracterización más amplia, las anomalías (de cualquier tipo) consisten en problemas empíricos que plantean dudas racionales sobre las credenciales empíricas de una teoría, independientemente de si otra teoría ha tenido éxito en su resolución.

Ya podemos dar una idea de lo que distingue las anomalías no refutatorias de los casos en que algunos fenómenos permanecen sin explicación por una teoría, ya sea porque caen fuera de su dominio de aplicación o porque, a pesar de estar incluidos en él, es previsible que la teoría, tal como está (esto es, con las mismas leyes), podrá explicarlos en el futuro. A diferencia del primer tipo de caso, las anomalías no refutatorias guardan una estrecha semejanza o conexión con ejemplos paradigmáticos de las aplicaciones intencionales de una teoría; a diferencia del segundo tipo de casos, estas anomalías, debido a su naturaleza recalcitrante, sugieren que la teoría necesitará modificaciones sustanciales, adiciones o incluso un reemplazo. Contra esto, y favoreciendo la visión de Laudan, podría argumentarse que a menos que exista una clase de contraste de teorías alternativas capaces de resolver ciertos problemas empíricos no refutatorios, no tenemos motivos para considerar a éstos últimos como anomalías en lugar de como meros problemas de ajuste entre la teoría y los datos, o, alternativamente, meros hallazgos empíricos contingentes, no considerados merecedores de explicación (incluso si son muy similares a los ejemplos paradigmáticos). En respuesta a la primera opción, debe enfatizarse que las anomalías no refutatorias no apuntan a aquellos casos en los que una teoría habla sólo de manera muy aproximada, sino a aquellos casos donde una

\footnotetext{
${ }^{1}$ Puede valer la pena recordar brevemente la noción kuhniana de anomalía, pues algunas de las ideas de Laudan ya habían sido sugeridas por Kuhn, quien sin embargo no se percató completamente de sus implicaciones para la concepción tradicional del apoyo evidencial. La noción general de anomalía introducida por Kuhn corresponde a aquellos problemas o fenómenos que una teoría no puede acomodar y que no se ajustan a las expectativas teóricas (1962/1970, 58). Tanto las anomalías refutatorias como las no refutatorias caen bajo la noción general anterior. Kuhn, a diferencia de Laudan, no considera que, para que una anomalía sea reconocida como tal, sea necesario que algún rival haya sido capaz de resolverla. Sostiene, por el contrario, que es la conciencia previa de la anomalía lo que inicia el proceso de modificación de teoría o cambio de teoría (1962/1970, 62).

${ }^{2}$ Cfr. Laudan, 1977, p. 29 (también n. 15). En consecuencia, Laudan compara lo que se ha llamado "las pérdidas de Kuhn" (Kuhn, 1962/1970, 107-108) con ciertas instancias de anomalías no refutatorias, a saber, aquellas en las que la teoría sucesora no proporciona ninguna explicación para los fenómenos que la teoría anterior ha cubierto.
} 
teoría, a pesar de los posibles esfuerzos en sentido contrario, permanece en silencio con respecto a ciertos fenómenos. En cuanto a la segunda posibilidad, es importante señalar que las anomalías consisten en fenómenos prominentes o llamativos que, dada su relevancia para la teoría, estos últimos deberían ser capaces de acomodar.

Pasemos ahora al aspecto más central y audaz de la caracterización laudaniana de las anomalías no refutatorias, a saber, la ampliación de la base evidencial o evaluativa de una teoría de modo que incluya afirmaciones empíricas verificadas que no están entre el conjunto de las consecuencias de la teoría ni entre el conjunto de las consecuencias excluidas por la teoría. En palabras de Laudan, su discusión de las anomalías no refutatorias implica el rechazo de "the consequentialist theory of evidence or plausibility" $(1995,28)$, así como el reconocimiento de que "(...) being a consequence of a hypothesis is neither necessary nor sufficient to qualify something as evidence for that hypothesis"(1995, 29). Las condiciones de justificación de una afirmación, por lo tanto, no deben equipararse con sus condiciones de verdad, ya que una limitada aplicabilidad de la teoría generaría dudas sobre su valor epistémico, independientemente de si sus condiciones de verdad están ampliamente satisfechas $(1995,33) .{ }^{3}$ La adecuación empírica de una teoría, entonces, no sólo depende de que las consecuencias empíricas de ésta sean verdaderas sino también de que correspondan a los fenómenos más llamativos de su dominio de aplicación. En términos modeloteóricos: la adecuación empírica de una teoría no sólo requiere que (en al menos uno de sus modelos) todas sus subestructuras empíricas sean isomórficas con los fenómenos correspondientes (van Fraassen, 1976), sino que también lo sean con todos los fenómenos en el dominio. El desafío, por tanto, consiste en caracterizar el tipo de información que, incluso si está lógicamente desconectada de lo que implica una teoría, no obstante proporciona evidencia crucial para la teoría y cae dentro de su dominio de aplicación.

Al ilustrar la noción de anomalía no refutatoria a partir de ciertos casos históricos, Laudan reitera dos ejemplos especialmente reveladores (1977, 29,

\footnotetext{
${ }^{3}$ En su artículo de 1988, T. Nickles argumenta que el modelo consecuencialista de la justificación científica debería combinarse con el modelo generativo de Laudan, ya que el segundo apunta a cambios teóricos que quedan fuera de la condicionalización estándar, donde se requiere que el conocimiento general permanezca fijo $(1988,10)$ Aunque en un contexto diferente, como es el campo de las matemáticas, I. Lakatos introdujo una noción similar a la de anomalía no refutatoria de Laudan, a saber, la de los falsadores heurísticos (heuristic falsifiers). Explica que, a diferencia de los falsificadores lógicos, que muestran que una teoría como tal es falsa (inconsistente), los falsificadores heurísticos simplemente muestran que una teoría no explica adecuadamente lo que se propuso explicar: es una teoría falsa del dominio informal en cuestión. En palabras del propio Lakatos: "the crucial role of heuristic refutations is to shift problems to more important ones, to stimulate the development of theoretical frameworks with more content" (Lakatos, 1967/1978, 40).
} 
2000, 167). ${ }^{4}$ El primero es el de la adición de la hipótesis nebular de Laplace a la mecánica newtoniana, que ilustra el caso en el que la teoría afectada por una anomalía no refutatoria se complementa con una especialización de la teoría (Brush, 1996, Laplace, 1796/1930). Un segundo ejemplo es el de la rivalidad entre la teoría contraccionista y la teoría de la deriva continental de Wegener (Laudan, 1977, 29 y 2000, 167, Cohen, 1985/2001), que desemboca en el rechazo de la teoría afectada por la anomalía no refutatoria y su sustitución por la teoría de las placas tectónicas, que incluye el postulado de la deriva continental.

El primero de los citados ejemplos se centra en la introducción, por parte de Laplace, de la hipótesis nebular para poder explicar el hecho de la unidireccionalidad y aproximada coplanaridad de los planetas (Laplace, 1796/1930, pp. 361-363). Este hecho constituía una anomalía no refutatoria para la mecánica newtoniana. Laplace, con todo, fue capaz de resolver la anomalía sin descartar la mecánica newtoniana, simplemente realizando un añadido informativo, a saber, la hipótesis nebular. Según esta hipótesis, el Sol era originalmente una gigantesca nube de gas rotando de manera uniforme. Debido al enfriamiento y a la acción de la gravedad, la nube se fue contrayendo y acelerando. La aceleración en la rotación causaría la separación de parte del borde de la masa en rotación, que acabaría por condensarse y formar un planeta. El proceso se iría repitiendo a lo largo del tiempo hasta llegar a darse la formación planetaria que contemplamos actualmente.

Los conceptos empíricos de la hipótesis nebular se encuentran determinados en su mayoría por postulados procedentes de la astronomía copernicana (como en el caso de los planetas y los satélites) y de la teoría del movimiento planetario de Kepler (como ocurre con el concepto de movimiento orbital). ${ }^{5}$ Teniendo en cuenta que la teoría de Kepler se apoya en el sistema copernicano y que la teoría de Kepler es a su vez aproximadamente reducible a la mecánica newtoniana (Balzer, Moulines \& Sneed, 1987, 374-383), las consideraciones anteriores implican que la mecánica newtoniana ya tenía los recursos conceptuales necesarios para representar hechos empíricos tales como la dirección y los movimientos relativos de los cuerpos celestes dentro del Sistema Solar. De hecho, la mecánica newtoniana no sólo tenía esos recursos sino también algunas aplicaciones intencionales paradigmáticas muy estrechamente rela-

\footnotetext{
${ }^{4}$ Un análisis de ambos puede encontrarse en Caamaño 2018a.

${ }^{5}$ Por supuesto, la hipótesis nebular también incluye conceptos de la mecánica newtoniana (como ocurre con el parámetro gravitacional estándar $\boldsymbol{\mu}$ ). Si consideramos la hipótesis nebular como una especialización de la mecánica newtoniana, entonces cada concepto teórico newtoniano sería también un concepto teórico de dicha hipótesis. Esto, sin embargo, no altera el punto principal que se enfatiza, a saber, que, sin las adiciones conceptuales de Laplace al marco newtoniano, este último carecería de los conceptos teóricos necesarios para explicar las anomalías no refutatorias, a pesar de tener los conceptos empíricos necesarios para describirlas.
} 
cionadas con tales tipos de fenómenos, como (cambios de) direccionalidad debido a colisiones o a la proximidad de cuerpos masivos, y (cambios de) forma debido a la distorsión ocasionada por las mareas. De hecho, la teoría de Laplace sólo agregó a las leyes de Newton algunas condiciones iniciales postuladas, así como una hipótesis sobre un proceso de contracción continuo. Vale la pena subrayar una vez más que, al buscar una explicación física de las anteriores características mecánicas del Sistema Solar, Laplace logró aplicar las leyes newtonianas a un dominio donde el propio Newton no creía que fueran aplicables. Donde este último vio una regularidad extraordinariamente armoniosa que requería una explicación teológica -algo explícito en su famosa Query 31-, el primero encontró otra llamativa regularidad abordable desde la mecánica newtoniana.

El segundo ejemplo frecuentemente empleado por Laudan se refiere a las anomalías no refutatorias involucradas en la revolución geológica, que acontece a mediados del siglo XX, y que es analizada en detalle por Paul Thagard (1992, cap. 7). Anomalías geológicas como el encaje entre las líneas costeras de los continentes permanecieron inexplicadas por las teorías contraccionistas y sólo se pudieron resolver una vez que la teoría fue rechazada y reemplazada por la teoría de las placas tectónicas, que incorpora la teoría de la deriva continental (Caamaño 2018b). El surgimiento de la teoría de las placas tectónicas (desarrollada entre 1962 y 1968) se produjo, precisamente, debido a toda una serie de anomalías no refutatorias que afectaban a las teorías geológicas existentes. De acuerdo con la literatura de referencia sobre el tema (Laudan, 1978, Cohen, 1985/2001, Thagard, 1992, Marvin 2001), algunas de las consecuencias más relevantes que entraña dicho cambio teórico tienen que ver con la definición del lecho marino y el supuesto de la distribución no azarosa de los terremotos. En ninguna de estas transiciones entre teorías geológicas se llegó a producir una refutación de lo establecido por las respectivas leyes de las teorías precedentes. El motor del cambio teórico fueron, principalmente, las anomalías no refutatorias para las que las teorías disponibles carecían de recursos explicativos suficientes, aunque sí de recursos descriptivos adecuados para describirlas como explananda.

Desde la teoría contraccionista, todavía vigente en geología hasta mediados del siglo XX, se postulaba el enfriamiento de la Tierra y, en consecuencia, la contracción de los continentes a lo largo del tiempo. La formación de montañas, tanto en los continentes como en los fondos marinos, se explicaba como un efecto del proceso de contracción. En la teoría de la deriva continental, por el contrario, se postulaba que todos los continentes que existen en la actualidad formaban inicialmente parte del mismo continente. El encaje actual del contorno de los continentes se explicaba a partir de una separación previa de los continentes, cuya ubicación habría cambiado a lo largo del tiem- 
po, lo que significa que los continentes se mueven unos con respecto otros. Finalmente, la formación de montañas se entendía como el resultado de cierta fricción cinética entre los continentes y los fondos marinos.

La teoría contraccionista y la de la deriva continental comparten muchos de sus conceptos empíricos, como el de corteza terrestre, continente, montaña o fondo marino. La determinación del ajuste entre los volúmenes de los continentes requiere asumir alguna teoría topológica y cartográfica, que se encontraría igualmente disponible a los partidarios de las teorías geológicas rivales. Además de los ya mencionados, éstas comparten algunos otros conceptos empíricos que se presuponen en una de las teorías y se incluyen explícitamente en la otra, como en el caso del ajuste entre los volúmenes de los continentes. Incluso si el concepto de ajuste entre volúmenes de continentes no se incluye explícitamente en la teoría contraccionista, se presupone como un concepto empírico, dado el concepto presupuesto de volumen y la inclusión del concepto de continente. El concepto de ajuste entre volúmenes de continentes puede caracterizarse pues independientemente de ambas teorías, simplemente teniendo en cuenta los estudios cartográficos pertinentes. Teniendo en cuenta que la forma, junto con la composición de material y la ubicación, siempre ha sido ampliamente considerada como una característica reveladora de los accidentes geológicos, el ajuste de la forma de los continentes difícilmente puede excluirse del dominio aplicativo de una teoría geológica general. Después de todo, la principal aplicación intencional de la teoría contraccionista, a saber, la formación de montañas, está determinada principalmente por características relacionadas con la forma. Por lo tanto, aquí encontramos de nuevo una característica esencial que conecta las aplicaciones intencionales de dos teorías sucesivas, una característica que, a pesar de sus peculiaridades llamativas, sólo se incluye entre las aplicaciones intencionales de una de las teorías. Dicho en pocas palabras, o bien el ajuste de los bordes continentales se descarta como un evento aleatorio, algo que parece altamente inverosímil, o debe reconocerse como parte del dominio de aplicación de una teoría geológica general.

\subsection{Superioridad de la predicción frente a la acomodación}

Todavía desde un punto de vista consecuencialista, pero tratando de afinar los recursos para evaluar la calidad del apoyo evidencial recibido por una teoría, Lipton y Forster son algunos de los autores que mantienen la superioridad epistémica de la capacidad predictiva frente a la capacidad para acomodar los fenómenos, indicando así otro factor clave para la evaluación del éxito en la adecuación empírica de las teorías. En efecto, la distinción de Forster entre contrastación diacrónica y sincrónica (Foster 2002, 233) coincide con la de Lipton entre predicción y acomodación (Lipton 1991/2004, 68). La contrastación diacrónica, al igual que la predicción, requeriría que las hipótesis se con- 
trastasen a partir de un conjunto de datos diferentes de aquellos empleados para construir la teoría. La contrastación sincrónica, en cambio, consistiría en la acomodación de los datos que se han empleado para elaborar la hipótesis. La superioridad de la predicción sobre la acomodación se debería a que, en la segunda, el éxito de la hipótesis para ajustarse a la evidencia sería atribuible a que la hipótesis había sido diseñada precisamente con ese fin, y no a una adecuación real con evidencias independientes del dominio de construcción de la teoría.

Aunque el predictivismo presenta ya un largo recorrido en la filosofía de la ciencia (Peirce 1931-58/1958, Whewell 1840, Duhem 1908/2015, Giere 1979/1984, Maher 1988, Lipton, 1991/2004, Mayo 1996, Foster, 2002, White 2003, Worrall 2014), la elucidación de la diferencia epistémica entre predicción y acomodación sigue dando lugar a controversias y a posiciones tanto predictivistas como anti-predictivistas (Barnes 2018, sección 7). Intuitivamente parece claro que la calidad de la evidencia proporcionada por las predicciones es mejor que la obtenida a través de la acomodación. En una primera aproximación se puede entenderse que esta diferencia en la calidad de la evidencia radica en la forma en que se diseña y pone a prueba una teoría predictiva, en contraposición con cómo se construiría y evaluaría una teoría "acomodaticia". En el primer caso, una concepción ad hoc de la teoría sería más difícil, ya que la evaluación de la teoría dependería de lo que establezca la teoría sobre eventos futuros o sobre eventos pasados que, incluso si ya se conocían, no fueron inicialmente abordados explicativamente por la teoría. Por el contrario, una teoría acomodaticia podría construirse sólo para encajar eventos ya incluidos en su dominio inicialmente orientado.

La explicación anterior de la diferencia entre predicción y acomodación, sin embargo, no es del todo correcta ni muy esclarecedora. No es del todo correcta porque presenta la acomodación como convergiendo con la adhocidad, cuando, en cierto sentido, la primera no implica necesariamente la última. La teoría darwiniana de la evolución, por ejemplo, pese a ser más acomodaticia que predictiva, no se considera una teoría ad hoc. La explicación intuitiva de la acomodación tampoco es muy aclaratoria, ya que, independientemente de cómo se haya concebido inicialmente una teoría (de manera ad hoc, acomodaticia o predictiva), puede resultar algo diferente en el futuro dependiendo de los diferentes aspectos dinámicos relacionados con la contrastación y evaluación teórica. Peter Lipton menciona la precisión, además de la falta de adhocidad, como las características más distintivas de las predicciones frente a las acomodaciones. Las implicaciones relativas a la adhocidad se discutirán en la siguiente sección, pero, en relación con la precisión, debe aclararse que una de las principales razones por las cuales las predicciones son precisas es que, a diferencia de las acomodaciones, suelen estar sujetas a un control experimental (Lipton, 1991/2004, 169). La anticipación de hechos desconocidos, por otro lado, incluso si constituye claramente una ventaja de las 
predicciones, no representa una de sus características más esenciales. No es sorprendente que el uso de la teoría de la relatividad general de Einstein para explicar la desviación del perihelio de Mercurio también se mencione como un ejemplo de predicción que proporciona un apoyo más fuerte que la mera acomodación, a pesar del hecho de que tal desviación era un fenómeno ya conocido. Dado que este uso laxo del término 'predicción' es habitual y genera cierta ambigüedad no deseada, aquí se empleará 'acomodación' en un sentido restringido, no solapado con la acepción laxa de 'predicción', la cual remitiría a una determinación precisa, no sólo de hechos futuros, sino también de hechos pasados (ya sean conocidos o desconocidos) mediante la aplicación de una teoría. En el sentido restringido que asumiré, al hablar de 'acomodación' me referiré a aquellos fenómenos conocidos que, o bien constituyeron los explananda originarios de la teoría, o bien, si no se encontraban entre tales explananda, una vez puestos en relación con la teoría, su determinación (explicación) inicial sólo se obtiene de forma imprecisa. Ciertamente, al optar por este uso de la terminología, asocio ya a la acomodación de hechos fuera del dominio de construcción de la teoría un carácter vago, no preciso que muchos de los autores críticos con el predictivismo no aceptarían, ya que parte de su crítica se apoya justamente en el carácter vago de ciertas predicciones y el carácter preciso de ciertas acomodaciones (Hitchcock \& Sober 2004). David Harker, otro autor crítico con el predictivismo, argumenta igualmente que el valor confirmatorio de ciertos datos empíricos depende de cómo ellos entren en juego mostrando la superioridad del contenido de la teoría, su fortaleza y simplicidad, con independencia de que dichos datos constituyan predicciones o acomodaciones (Harker 2008). Sin embargo, como ha puesto de relieve Deborah Mayo en su defensa de la conexión entre contrastación severa y predicción (1996, capítulo 9), la construcción (o reconstrucción) de la hipótesis tras disponer de la prueba empírica es típico de la acomodación frente a la predicción, implicando ésta efectivamente una riqueza de contenido de la teoría suficiente como para determinar con precisión el hecho empírico antes de que se conozca o sin que se haya diseñado la teoría a tal fin. Por otra parte, hablar de predicciones imprecisas, parece forzar el concepto de "predición" hasta dar cabida a casos en los resultaría muy cuestionable la aplicabilidad de dicho concepto (pensemos en las "predicciones" de los horóscopos o casos similares, Díez \& Moulines 1999, 86-7).

A diferencia de lo que sucede con las teorías predictivas, pues, en el caso de las teorías acomodaticias, el dominio de los fenómenos que impulsaron la construcción, modificación o compleción de una teoría no es diferente del dominio de los fenómenos que proporciona la base evidencial para contrastar la teoría. Esto sugiere, como condición mínima para el éxito empírico de una teoría, que su dominio evidencial o evaluativo sea diferente de su dominio de "construcción" (Maher 1988, Lipton, 1991/2004, Worrall 2014). Esto, a su vez, implicaría una mayor independencia de la evidencia con respecto a la 
teoría para la cual desempeña el papel evidencial, en el sentido de que la evidencia involucrada en la contrastación de la teoría no sería parte del dominio empírico al que la teoría pretendía aplicarse. En la medida en que lo importante es preservar el carácter independiente de la evidencia (algo especialmente puesto de relieve por Peirce) y no necesariamente el descubrimiento de hechos nuevos, los principales fenómenos invocados en la justificación de una teoría no deberían formar parte del contenido inicial de la misma. Como subraya White, una teoría está peor confirmada por cierto dato en la medida en que la teoría fue diseñada para dar cuenta de él (White 2003, 656), como considera que ocurre en el caso de las teorías que sólo poseen capacidad de acomodación.

Aquellos autores críticos con el predictivismo, además de esgrimir ejemplos de teorías acomodaticias explicativamente valiosas y de teorías predictivas explicativamente desacertadas, cuestionan, por transitoria, la relevancia epistémica del factor predictivo. Destacan, en cambio, la importancia de las probabilidades iniciales atribuidas a las teorías en competencia, que aumentarían con los éxitos empíricos pasados, con independencia de que éstos se den en forma de predicciones o acomodaciones (Barnes 2018). Este tipo de argumento, sin embargo, obvia aquellos rasgos de las predicciones sobre los que aquí se ha hecho hincapié y que conllevan una superioridad en la calidad de la evidencia que ellas proporcionan. Los datos predichos, a diferencia de los acomodados, no se utilizan en la formulación de la teoría para los que cuentan como evidencia, es decir, preservan su independencia, suelen ser más susceptibles de control experimental y de determinación precisa en virtud del contenido extra de la teoría. En pocas palabras, podría decirse que la capacidad predictiva manifiesta la riqueza informativa de una teoría, dado que es dicha riqueza la que posibilita determinar ciertos datos empíricos no contemplados en el desarrollo de la teoría. Todos estos rasgos confieren una mayor fuerza confirmatoria a la evidencia con respecto a la teoría que se evalúa.

\subsection{El uso limitado de hipótesis ad hoc}

Veamos, a continuación, otra condición para la adecuación empírica, como es el uso limitado de hipótesis ad hoc, es decir, hipótesis auxiliares no corroboradas. P. Thagard ha establecido esta condición como uno de los criterios de simplicidad para la elección de teorías (Thagard, 1978, 86-89), sin embargo, en la medida en que la condición se refiere esencialmente a la corroboración, también puede entenderse como una condición para determinar de forma más rigurosa la adecuación empírica de una teoría De acuerdo con este autor: 
An auxiliary hypothesis is a statement, not part of the original theory, which is assumed in order to help explain one element of $F$ [the facts] or a small fraction of the elements of $F$. (cursiva en el original, Thagard, 1978, 86)

Antes de realizar esta caracterización, Thagard ya ha descartado la posibilidad de que $A$ (es decir, un conjunto de hipótesis auxiliares) se equipare con $C$, es decir, un conjunto de condiciones no problemáticas aceptadas independientemente de $T$ o $F$ y asumidas en la aplicación de $T$. $A$, por el contrario, incluiría supuestos con una aplicación limitada, restringida, en su uso, a una clase de hecho. Teniendo en cuenta lo visto en la anterior sección, podríamos afirmar que se trataría de una acomodación teórica con un alcance extremadamente limitado, esto es, no generalizable. Como ejemplos, Thagard menciona la suposición, introducida por Huygens para explicar la refracción irregular en el cristal de Islandia, de que algunas ondas son esferoidales más que esféricas, o su suposición de que la velocidad de la luz es más lenta en los medios más densos para explicar la ley de refracción de Snell. Thagard señala que estas suposiciones a veces coinciden con hipótesis $a d h o c$, aunque señala que, dado que la adhocidad es una propiedad dinámica, los supuestos auxiliares pueden perder este estado ad hoc en algún momento. Esto puede ocurrir, ya porque llegan a confirmarse, ya debido a que las teorías que compiten comparten esas mismas suposiciones.

Después de examinar minuciosamente las descripciones más comunes de adhocidad, tanto J. Leplin como M. Carrier han argumentado de forma independiente que ninguna de estas explicaciones enfatiza suficientemente la característica verdaderamente distintiva de las hipótesis ad hoc. Ambos coinciden en que el carácter ad hoc de una hipótesis no está determinado por la forma en que fue concebida, sino más bien por el hecho de que no recibe apoyo empírico independiente. Los dos autores convergen al señalar que comportar un contenido empírico extra constituye un requisito clave para evitar la adhocidad. En palabras de Carrier:

A hypothesis explains a fact in a non-ad-hoc manner, if it simultaneously explains at least one additional independent fact that either constitutes an anomaly for the rival theory or that falls beyond its realm of application, i. e. that is neither derivable from nor inconsistent with the competing approach. (Carrier, 1988, 206).

Aunque implica algunos requisitos adicionales, la definición de adhocidad de J. Leplin también incluye una condición relativa a la falta de apoyo empírico independiente (Leplin 1975, 336-337). Sin embargo, su contribución más novedosa al análisis de la adhocidad consiste en una condición concerniente a la no fundamentalidad. Esta condición revela algunos aspectos importantes 
relacionados con la adhocidad, como la naturaleza holística local de las anomalías y el correspondiente requisito de que cada hipótesis permita resolver varias anomalías juntas. La condición conlleva la presuposición de que las teorías no fundamentales, es decir, aquellas con una gran variedad de insuficiencias graves, son las únicas afectadas por una adhocidad recalcitrante. Dada la naturaleza dinámica de la justificación de teorías, que, como reconocen Leplin y Carrier, convierte la adhocidad en una propiedad dinámica e inestable, también es posible (aunque improbable) que una teoría no fundamental supere sus dificultades y se convierta en fundamental. Por lo tanto, la distinción de Leplin entre teorías incompletas (donde finalmente las hipótesis ad hoc se convierten en no ad-hoc) y teorías no fundamentales (donde se descarta la posibilidad anterior) debe considerarse con cierta cautela. Por un lado, nunca podemos estar seguros de que una teoría incompleta no vaya a revelarse como no fundamental ni, a la inversa, de que una no fundamental se revele como lo contrario. Esa es la razón por la cual, con frecuencia, los juicios unánimes sobre si una teoría es de uno u otro tipo sólo se emiten cuando la teoría ha sido completada o reemplazada, y, no obstante, sí se llegan a emitir juicios unánimes sobre la adhocidad antes de que la compleción o el reemplazo ocurran. A pesar de todo, la no fundamentalidad sí resulta relevante al apuntar a la estrecha conexión que debería mantenerse entre el carácter holista de las anomalías y el carácter holista de su explicación teórica.

\section{Bosquejo de un criterio de adecuación empírica}

A partir del análisis previo, es posible elaborar el esquema de un criterio para el éxito empírico de las teorías. Los tres requisitos cualitativos para el éxito teórico comentados anteriormente tienen algo en común: implican algunas condiciones relativas a la relación entre una teoría y subconjuntos diferentes de su dominio de aplicación. Por ello, antes de delinear un criterio de adecuación empírica que incluya los requisitos anteriores, se deben trazar varias distinciones entre diferentes subdominios de una teoría. Hay dos divisiones básicas: una entre aplicaciones exitosas y no exitosas, y la otra entre el dominio de construcción y el dominio evaluativo. En éste último, se podrían diferenciar cuatro subdominios: dos que corresponden a anomalías refutatorias y no refutatorias, por un lado, y dos (ortogonales con los anteriores) correspondientes a predicciones y acomodaciones exitosas, por el otro. En aras de la simplicidad, haré uso de las siguientes convenciones notacionales, todas ellas referentes a las especificaciones del dominio evaluativo de las teorías: 
$\mathrm{E}=$ dominio evaluativo

$\mathrm{E}_{\mathrm{S}}=$ aplicaciones exitosas $\quad \mathrm{E}_{\mathrm{U}}=$ aplicaciones no exitosas

$\mathrm{E}_{\mathrm{P}}=$ predicciones exitosas $\quad \mathrm{E}_{\mathrm{R}}=$ anomalías refutatorias

$\mathrm{E}_{\mathrm{A}}=$ acomodaciones exitosas $\quad \mathrm{E}_{\mathrm{N}}=$ anomalías no refutatorias

$\mathrm{E}_{\mathrm{Ab}}=$ acomodaciones ad hoc

El criterio empírico de determinación del éxito empírico de una teoría puede presentarse esquemáticamente del siguiente modo:

$\mathrm{T}_{2}$ es empíricamente más exitoso que $\mathrm{T}_{1}$ si se cumplen las siguientes condiciones:

a) $\mathrm{E}_{\mathrm{S}}\left(\mathrm{T}_{1}\right) \subseteq \mathrm{E}_{\mathrm{S}}\left(\mathrm{T}_{2}\right)$,

b) $\left\|\mathrm{E}_{\mathrm{U}}\left(\mathrm{T}_{2}\right)\right\|<\left\|\mathrm{E}_{\mathrm{U}}\left(\mathrm{T}_{1}\right)\right\|$,

c) $\mathrm{E}_{\mathrm{N}}\left(\mathrm{T}_{1}\right) \cap \mathrm{E}_{\mathrm{S}}\left(\mathrm{T}_{2}\right) \neq \varnothing$,

d) $\mathrm{E}_{\mathrm{A}}\left(\mathrm{T}_{1}\right) \cap \mathrm{E}_{\mathrm{P}}\left(\mathrm{T}_{2}\right) \neq \varnothing$,

e) $\left\|\mathrm{E}_{\mathrm{Ad}}\left(\mathrm{T}_{2}\right)\right\|<\left\|\mathrm{E}_{\mathrm{Ab}}\left(\mathrm{T}_{1}\right)\right\|$.

Téngase en cuenta que estas cuatro condiciones se presentan aquí como globalmente suficientes para el éxito empírico comparativo, pero no como globalmente necesarias, lo que revelaría un requisito demasiado fuerte para la mayoría de los casos de elección teórica. Extrapolando una idea que John L. Mackie aplica en su caracterización de las causas, se podría decir que el criterio formulado arriba se establece a partir de condiciones tipo INUS ("Insufficient but Necessary parts of a condition which is itself Unnecessary but Sufficient", Mackie 1965). Las condiciones a)-b) reproducen los criterios cuantitativos tradicionales, conforme a los cuales la teoría empíricamente superior $\left(\mathrm{T}_{2}\right)$ ha de incluir, entre sus aplicaciones exitosas, las aplicaciones exitosas de la teoría rival $\left(\mathrm{T}_{1}\right)$, teniendo, en cambio, menos aplicaciones fallidas. Las condiciones c)-e) son más sensibles a los aspectos cualitativos destacados en este trabajo al exigir: primero, que alguna anomalía no refutatoria de $\mathrm{T}_{1}$ constituya una aplicación exitosa de $\mathrm{T}_{2}$, segundo, que algunas acomodaciones en $\mathrm{T}_{1}$ constituyan predicciones en $\mathrm{T}_{2}, \mathrm{y}$, tercero, que $\mathrm{T}_{2}$ incluya menos hipótesis ad hoc que $\mathrm{T}_{1}$. Nótese que sólo en los casos de adaptaciones ad hoc el dominio de construcción y el dominio evaluativo se superponen por completo. Las anomalías no refutatorias de una teoría, por otro lado, siempre están incluidas dentro del subdominio formado por sus aplicaciones no exitosas. 
Este enfoque del éxito teórico puede verse como un complemento de la compleja matriz de evaluación de Kuipers (2005), al agregar algunos requisitos cualitativos que no recoge su propuesta. La triple evaluación de las teorías presentada por él implica la consideración de lo que llama "resultados neutrales", que corresponden a las anomalías no refutatorias de Laudan y representan un parámetro importante en la evaluación comparativa de éxito teórico. En sus modelos simétricos para el método hipotético-deductivo de evaluación independiente de teorías, es decir, sus modelos micro y macro, para que una teoría sea al menos tan exitosa como la anterior, algunas condiciones generales de adecuación deben ser satisfechas no sólo por las definiciones de "éxito" y "problema", pero también para la de "resultado neutral", que equivale a la noción de anomalía no refutatoria. Contrariamente a lo sucedido en los modelos asimétricos, donde las condiciones de éxito sólo se refieren explícitamente a problemas individuales y éxitos generales, mientras que los resultados neutrales permanecen ocultos, en el modelo simétrico, estos últimos juegan un papel importante (Kuipers 2005, 52). Al tener explícitamente en cuenta los tres tipos de resultados, los modelos simétricos de Kuiper cumplen con los requisitos evaluativos de Laudan en relación con el alcance más amplio de las teorías más exitosas. Teniendo en cuenta que los hechos generales neutrales para una teoría no constituyen ni un problema ni un éxito, una teoría exitosa debería transformar los problemas generales en hechos neutrales y los hechos generales neutrales en éxitos. El mismo tipo de requisito debe cumplirse en el nivel micro para éxitos individuales, problemas individuales y resultados neutrales. Las instancias neutrales también pueden permanecer neutrales o transformarse en éxito.

Como se señaló anteriormente, el análisis detallado desarrollado por Kuipers no incorpora algunos parámetros cualitativos, como el carácter ad hoc y la mera capacidad de acomodación, que resultan esenciales en la evaluación del éxito empírico de las teorías.

\section{Conclusión}

En síntesis, podría decirse que las concepciones tradicionales de la adecuación empírica se han revelado en un sentido demasiado amplio y en otro demasiado estrecho. Lo primero se hace evidente en el descuido de los problemas relativos a la adhocidad y la acomodación, el segundo en la atención insuficiente prestada a cuestiones relativas a la apertura aplicativa o incompletitud de una teoría. Todas estas cuestiones, sin embargo, parecen cruciales para una evaluación comparativa del éxito empírico de las teorías. Los problemas relativos a la acomodación y al carácter ad hoc requerirían un análisis del nivel de precisión de una teoría, de su inclusión de contenido empírico extra con respecto a su dominio de construcción y de su aplicabilidad holista a anomalías de carácter holista. Las cuestiones relativas a la estrechez del al- 
cance requerirían el reconocimiento de anomalías no refutatorias como parte del dominio evaluativo de una teoría. Los factores anteriores deben tenerse en cuenta si se quiere evaluar el éxito empírico de una teoría en toda su complejidad.

\section{Referencias bibliográficas}

Balzer, Wolfgang, Moulines, Carlos U., Sneed, Joseph D. (1987). An Architectonic for Science. The Structuralist Program. Dordrecht: Reidel.

Barnes, Eric C. (2018). "Prediction versus Accommodation", The Stanford Encyclopedia of Philosophy, Edward N. Zalta (ed.), forthcoming URL: https://plato.stanford.edu/archives/fall2018/entries/prediction-accommodation/.

Brush, Stephen G. (1996). Nebulous Earth: The Origin of the Solar System and the Core of the Earth from Laplace to Jeffreys. Cambridge: CUP.

Caamaño Alegre, María (2018a). "Making Sense of Non-Refuting Anomalies", Journal for General Philosophy of Science, DOI 10.1007/s10838018-9409-0, 2018.

Caamaño Alegre, María (2018b). "Drift Theory and Plate Tectonics: A Case of Embedding in Geology", Foundations of Science, 23, n.1, marzo, 2018, 17-35.

Carrier, Martin (1988). "On Novel Facts. A Discussion of Criteria for Nonad-hoc-ness in the Methodology of Scientific Research Programmes", Zeitschrift für allgemeine Wissenschaftstheorie, 19, 2, 205-231.

Cohen, I. Bernard (1985/2001). "Continental Drift and Plate Tectonics: A Revolution in the Earth Science", Revolution in Science. Cambridge, MA: Harvard University Press, 29: 446-466.

Díez, José A., Moulines, C. Ulises (1999). Fundamentos de Filosofía de la Ciencia. Barcelona: Ariel.

Duhem, Pierre (1908/2015). To Save the Phenomena. An Essay on the Idea of Physical Theory From Plato To Galileo. Chicago: The University of Chicago Press.

Forster, Malcolm R. (2002). "Hard Problems in the Philosophy of Science: Idealisation and Commensurability", in R. Nola \& H. Sankey (ed.), After Popper, Kuhn and Feyerabend. Recent Issues in Theories of Scientific Method. Dordrecht: Kluwer Academic Publishers, pp. 231-250.

Giere, Ronald N. (1979/1984). Understanding Scientific Reasoning, second edition. New York: Holt, Rinehart, and Winston. 
Harker, David (2008). "On the Predilections for Predictions", British Journal for the Philosophy of Science, 59(3): 429-453.

Hitchcock, Christopher, Sober, Elliott (2004). "Prediction versus Accommodation and the Risk of Overfitting", British Journal for the Philosophy of Science, 55(1): 1-34.

Kuhn, Thomas S. (1962/1970). The Structure of Scientific Revolutions. Chicago: The University of Chicago Press.

Kuipers, Theo A. F. (2005). "The Threefold Evaluation of Theories. A Synopsis of From Instrumentalism To Constructive Realism. On Some Relations Between Confirmation, Empirical Progress, and Truth Approximation (2000)", in R. Festa, A. Aliseda \& J. Peijnenburg (eds.), Confirmation, Empirical Progress, and Truth Approximation (Poznan Studies in the Philosophy of the Sciences and the Humanities, vol. 83). Amsterdam/New York, NY: Rodopi, 23-85.

Lakatos, Irme (1967/1978). Mathematics, Science and Epistemology (Philosophical Papers Vol. II), ed. J. Worrall and G. Currie. Cambridge: Cambridge University Press.

Laudan, Larry (1977). Progress and its problems. Berkeley: UCP.

Laudan, Larry (1981). "Anomalous Anomalies", Philosophy of Science, vol. 48, n. 4: 618-619.

Laudan, Larry (1995). "Damn the Consequences!", Proceedings and Addresses of the APA, 69, 27-34.

Laudan, Larry (2000). "Is Epistemology Adequate to the Task of Rational Theory Evaluation?", en R. Nola \& H. Sankey (ed.), After Popper, Kuhn and Feyerabend. Dordrecht: Kluwer Academic Publishers: 165-175.

Laudan, Rachel (1978). "The Recent Revolution in Geology and Kuhn's Theory of Scientific Change", PSA: Proceedings of the Biennial Meeting of the Philosophy of Science Association, vol. 2, Symposia and Invited Papers: 227-239.

Leplin, Jarrett (1975). "The Concept of an Ad Hoc Hypothesis", Studies in History and Philosophy of Science, 5, 309-345.

Lipton, Peter (1991/2004). Inference to the Best Explanation. New York: Routledge, 2004.

Mackie, John L. (1965). "Causes and Conditions", American Philosophical Quarterly, 12: 245-65. 
Maher, Patrick (1988). "Prediction, Accommodation, and the Logic of Discovery", PSA: Proceedings of the Biennial Meeting of the Philosophy of Science Association 1988, 1: 273-285.

Mayo, Deborah G. (1996). Error and the Growth of Experimental Knowledge. Chicago and London: University of Chicago Press.

Moulines, Carlos U. (2000). "Is There Genuinely Scientific Progress?", en Adam Jonkisz \& Leon Koj (eds.), On Comparing and Evaluating Scientific Theories, Poznan Studies in the Philosophy of the Sciences and the Humanities, 72: 173-197.

Nickles, Thomas (1988). "Truth or Consequences? Generative versus Consequential Justification in Science", PSA, 2, 393-405.

Peirce, Charles S. (1931-58/1958). Collected Papers of Charles Sanders Peirce, Charles Hartshorne, Paul Weiss \& Arthur W. Burks (eds.), Cambridge. Massachusetts: Harvard University Press.

Popper, K. R. (1962/1965). "Truth, Rationality and the Growth of Scientific Knowledge", en Conjectures and Refutations. New York: Harper and Row, 215-252.

Thagard, Paul R. (1978). "The Best Explanation: Criterion for Theory Choice”, The Journal of Philosophy, Vol. 75, No. 2, Feb., 76-92.

Thagard, Paul R. (1992). Conceptual Revolutions. Princeton University Press, Princeton.

Van Fraassen, Bas (1976). "To Save the Phenomena”, en Richard Boyd, Philip Gasper, \& J. D. Trout (eds.), The Philosophy of Science, Cambridge: Massachusetts Institute of Technology, 1992, 187-194.

Whewell, William (1840). The Philosophy of the Inductive Sciences, Founded Upon Their History. London: John W. Parker.

White, Roger (2003). "The Epistemic Advantage of Prediction Over Accommodation", Mind, 112 (448):653-683.

Worrall, John (2014). "Prediction and Accommodation Revisited", Studies in History and Philosophy of Science Part A, 45: 54-61. 